\title{
A Comparative Analysis of Artificial Immune Network Models
}

\author{
Juan Carlos Galeano \\ jcgaleanoh@unal.edu.co \\ Angélica Veloza-Suan \\ Fabio A. González \\ avelozas@unal.edu.co fagonzalezo@unal.edu.co \\ Lab. de Investigación en Sistemas Inteligentes \\ Dept. de Ingeniería de Sistemas e Industrial \\ Universidad Nacional de Colombia \\ Bogotá, Colombia
}

\begin{abstract}
This paper presents a review of different artificial immune network models, which have been published during the last years. A general model of artificial immune network is presented, which provides a common notation that allows the comparison of different models. A descriptive and comparative analysis is presented emphasizing similarities, differences and relationships between models. Finally, some conclusions and suggestions for improving existent models are presented.
\end{abstract}

\section{Categories and Subject Descriptors}

A.1 [Introductory and Survey]

; I.2.8 [Artificial Intelligence]: Problem Solving, Control Methods, and Search-Heuristic methods, Artificial immune systems

; I.5.1 [Pattern Recongnition]: Models

\section{General Terms}

Algorithms

\section{Keywords}

Artificial Immune System, Artificial Immune Network

\section{INTRODUCTION}

Since Ishiguro published the first Artificial Immune Network (AIN) computational model in 1994 [11], several new AIN models have been proposed to solve different kind of problems such as clustering, data analysis, and classification. Nowadays, AINs have become a well established area of research in the field of artificial immune systems.

Even though all the models are based on Jerne's Immune Network Theory [13], they present differences in terms of

Permission to make digital or hard copies of all or part of this work for personal or classroom use is granted without fee provided that copies are not made or distributed for profit or commercial advantage and that copies bear this notice and the full citation on the first page. To copy otherwise, to republish, to post on servers or to redistribute to lists, requires prior specific permission and/or a fee.

GECCO'05, June 25-29, 2005, Washington, DC, USA.

Copyright 2005 ACM 1-59593-010-8/05/0006 ...\$5.00. what mechanisms are modeled, how they are modeled, the type of representation used, and the intended application. The goal of this paper is to make a descriptive and comparative analysis of the most relevant AIN models that have been published to this date. The models are classified according to how some of them evolved from other ones. A general AIN model is introduced, providing a framework to discuss the coincidences and differences of the reviewed models. The main aim of this work is to provide elements to understand the current state of development of AIN research and to give useful insights for future work.

The rest of the paper is organized as follows: Section 2 presents a brief review of the main concepts and definitions from immune network theory; Section 3 introduces a general model of AIN, and describes different AIN models taking into account their structure, dynamics and meta-dynamics; finally, Section 4 presents the conclusions and some suggestions for improving existent models.

\section{IMMUNE NETWORK THEORY}

The immune network theory was proposed by Jerne [13] as a way to explain the memory and learning capabilities exhibited by the immune system. The principal hypothesis of this theory states that immune memory is maintained by B-cells interacting with each other, even in the absence of foreign antigens. These interactions can be either excitatory or inhibitory. The production of a given antibody (elicited by an external antigen) stimulates/suppresses the production of other antibodies that stimulate/suppress the production of other antibodies and so on [22]. Notice that the word antigen denotes those molecules that the immune cells/molecules are able to recognize, thus it is necessary to differentiate between self antigens (antibodies) and non-self antigens. Accordingly with the notation suggested by Jerne [13], the portion on the antigen's surface that an antibody recognizes is named epitope, the portion used by an antibody to recognize antigens is named paratope, and the epitope of an antibody (self antigen) is named idiotope.

Based on Jerne's work, some models of immune network were developed using differential equations to predict the antibody concentration during and after an immune response. The first models were proposed by Jerne, Farmer et al., and Varela et al., see [5] for a summary.

An important concept for immune network modeling was introduced by Perelson [22], the shape-space. This concept 
considers antibody and antigen shapes as points in an ndimensional space, the shape-space, where each dimension is related to some principal characteristic involved in the recognition process. Antibodies recognize those antigens within the scope defined by a sphere centered at the antibody with a certain recognition radius.

\section{ARTIFICIAL IMMUNE NETWORKS}

An Artificial Immune Network (AIN) is a bio-inspired computational model that uses ideas and concepts from the immune network theory, mainly the interactions among Bcells (stimulation and suppression), and the cloning and mutation process. Several models have been proposed for problem solving in areas such as data analysis, pattern recognition, autonomous navigation and function optimization. These models will be reviewed in Subsection 3.2 and compared in Subsection 3.3.

\subsection{A General Model of AIN (GAIN)}

In this section, a general training algorithm for AIN is presented, which reflects the general steps common to the different reviewed AIN models, and introduces a mathematical notation, which allows their comparison. The algorithm is described in Figure 1. Other AIN general algorithms have been presented before by de Castro [3] and Garret [9].

The algorithm receives as input a set of antigens (represented by the set $A$ ), which are going to be presented to the network, and returns an immune network composed of a set of B-cells and connections between them. Notice that some AIN models do not represent explicitly the network structure.

The first step is to create an initial set of B-cells (represented by the set $B$ ). Some models use a subset of the antigen set and others generate the B-cells in a random fashion. After this, an iterative process is performed starting by presenting the set of antigens to the network. For each antigen and each B-cell the stimulation is calculated. This is represented by the following function:

$$
f_{\text {stimulation }}^{A}: A \times B \rightarrow \Re \text {. }
$$

In most of models the stimulation is a function of an affinity measure, which is defined in the space where B-cells and antigens are represented. In such a case, the stimulation measure is defined as follows:

$$
f_{\text {stimulation }}^{A}(a, b):=g\left(f_{\text {affinity }}(a, b)\right),
$$

where $f_{\text {affinity }}: B \cup A \times B \cup A \rightarrow \Re$ and $g: \Re \rightarrow \Re$. $f_{\text {affinity }}$ measures the similarity/complementarity between elements in the shape-space. In many cases, $f_{\text {affinity }}$ corresponds to a metric or pseudo-metric in the shape-space. $g$ calculates the amount of stimulation produced by an antigen with a given affinity with the B-cell.

In the next step B-cells are allowed to interact with each other, this is done by calculating the stimulation and suppression effects between them. These effects are represented by the following functions:

$$
f_{\text {stimulation }}^{B}: B \times B \rightarrow \Re \text { and } f_{\text {suppression }}^{B}: B \times B \rightarrow \Re
$$

Similar to antigen/B-cell stimulation, B-cell/B-cell stimulation (and suppression) could be calculated as a function of B-cell/B-cell affinity. Total stimulation $F: B \rightarrow \Re$ of
B-cells is calculated by summing up the effects caused by antigen and network interactions :

$$
\begin{aligned}
F(b)= & \sum_{a \in A} f_{\text {stimulation }}^{A}(a, b) \\
& +\sum_{b^{\prime} \in B, b^{\prime} \neq b} f_{\text {stimulation }}^{B}\left(b^{\prime}, b\right) \\
& +\sum_{b^{\prime} \in B, b^{\prime} \neq b} f_{\text {suppression }}^{B}\left(b^{\prime}, b\right), b \in B .
\end{aligned}
$$

Based on total stimulation, some B-cells are selected and $f_{\text {cloning }}(b)$ copies of each selected B-cell $b$ are created. Those copies undergo mutation with a certain mutation rate. Some models interpret this rate as the probability of a B-cell to be selected for suffering mutation; other models interpret it as the proportion of the B-cell fields that will be changed. In the metadynamics step, some useless B-cells are removed from the network, new B-cells are created randomly and incorporated into the network, and links among all B-cells are reorganized.

Finally, when the stopping criterion is met, the current network is returned.

\subsection{A Review of AIN Models}

Many of the published AIN models are variations of previously proposed models. This generates a dependency relationship between models, which could be represented as a genealogical tree. Such a tree is presented in Figure 2. All the AIN models in the first level can be considered as computational versions of Jerne's, Farmer's and Varela and Couthino's models placed in the tree's root. Those models have experimented some variations or have inspired other models which are presented in the lower levels.

A model is either a modification or is based on its parent model. Each branch of the tree will be described taking into account the differences and similarities between models, structure, dynamics and metadynamics [5] of the models in that branch, as well as the intended application. The structure refers to the interactions among network elements, the network dynamics refers to the mechanisms employed by the network to adapt to itself and to the environment, and the metadynamics describes the methods for adding new elements to the network and removing useless elements from the network [5].

\subsubsection{The Hunt \& Cooke Branch}

In 1996 Hunt and Cooke [10] proposed an artificial immune network, which was applied to pattern recognition tasks in DNA sequences. That model considered the immune system as a network of B-cells that are related to other B-cells by its affinity and its enmity. Such a relationships are based on Hamming distance following the Farmer's work [8]. Any B-cell undergoes cloning and mutation, if the current non-self antigen makes it stimulated enough, this is, if the B-cell stimulation level is greater than a threshold. The cloning process produces a number of exact copies of the B-cells depending on the stimulation level. The mutation process is based on a random selection between three kind of techniques. At the end of each iteration a subpopulation of the less stimulated B-cells are removed from the network and the same percentage of new cells is generated and incorporated in.

AINE (Artificial Immune NEtwork): Timmis et al. [25] proposed in 2000 an AIN that represents an adaptation 
$\mathbf{G A I N}(A$ : antigen set)

1: initialization

1.1: assign $B$ an initial set of B-cells

1.2: initialize network structure $L$

2: repeat until a stop criterion is met

2.1: antigen presentation:

$\triangleright$ Antigen/B-cell affinity

2.1.1: calculate $f_{\text {affinity }}(a, b)$ for all $a \in A, b \in B$

$\triangleright$ Antigen/B-cell stimulation

2.1.2: calculate $f_{\text {stimulation }}^{A}(b, a)$ for all $a \in A$ and $b \in B$

2.2: B-cell interaction:

$\triangleright B$-cell/B-cell stimulation/suppression

2.2.1: calculate $f_{\text {stimulation }}^{B}\left(b^{\prime}, b\right)$ and $f_{\text {suppression }}^{B}\left(b^{\prime}, b\right)$ for all $b, b^{\prime} \in B$

2.3: affinity maturation:

$\triangleright$ Total stimulation

2.3.1: calculate $F(b):=\sum_{a \in A, b^{\prime} \in B, b^{\prime} \neq b} f_{\text {stimulation }}^{A}(a, b)+f_{\text {stimulation }}^{B}\left(b^{\prime}, b\right)+f_{\text {suppression }}^{B}\left(b^{\prime}, b\right), b \in B$

2.3.2: create $f_{\text {cloning }}(b)$ clones of the B-cell $b$ and mutate them

2.3.3: calculate stimulation of all new B-cells

2.4: metadynamics:

$\triangleright$ deletion/creation of B-cells and links

2.4.1: update network structure $L$

$\triangleright$ Return immune network

3: $\operatorname{return}(B, L)$

Figure 1: A General Artificial Immune Network algorithm.

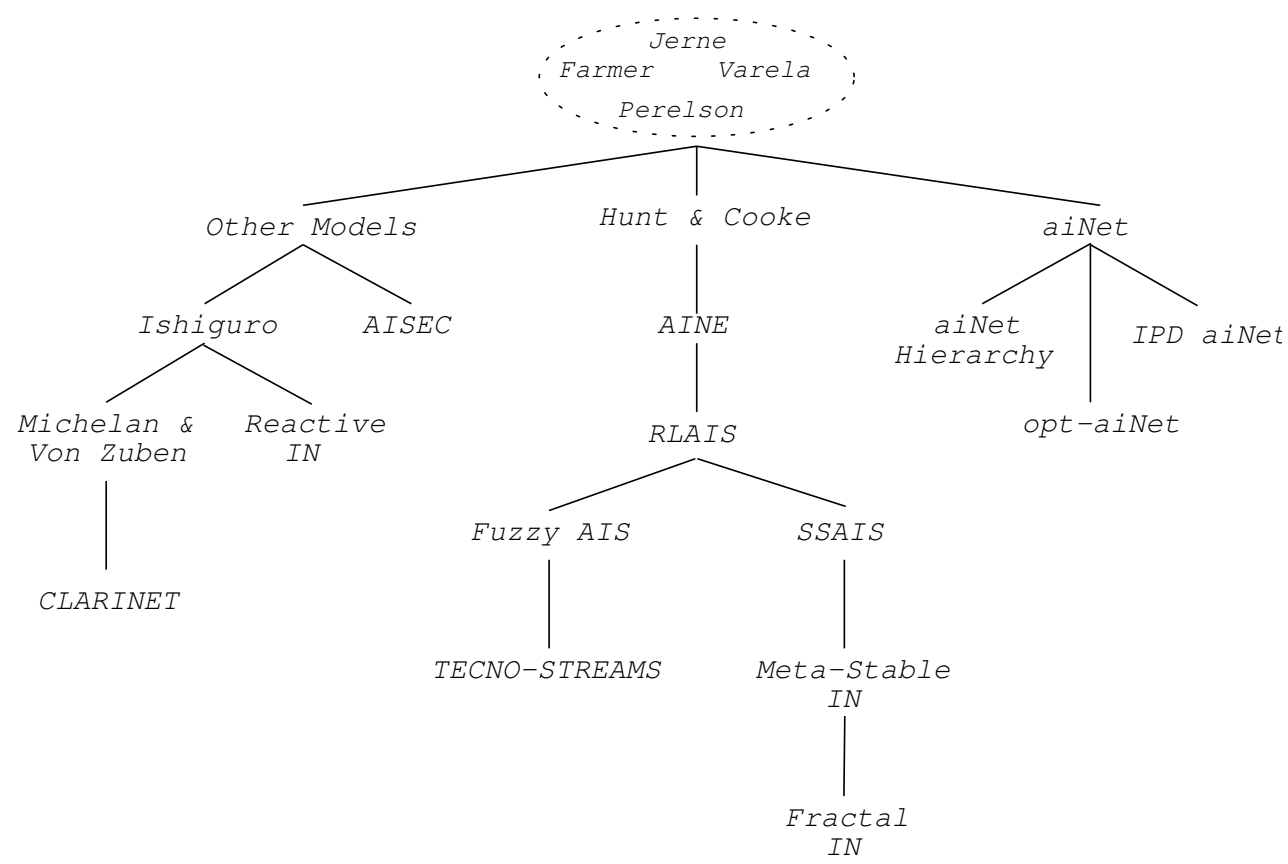

Figure 2: Genealogical Tree of AIN Models: each model is a modification or is based on its parent. 
of Hunt and Cooke's model to perform data analysis tasks. B-cells are related to each other by stimulation and suppression resembling the affinity and enmity interactions of Hunt and Cooke's model. Those cells with stimulation level greater than a threshold undergo cloning proportionally to their stimulation level. The mutation method assigns a fix probability for each field in the clones to be mutated. This method corresponds to one of the three mutation methods used by the Hunt and Cooke's model. At the end of each iteration, the $5 \%$ of the weakest, less stimulated, B-cells are removed from the network. Guided for the data analysis application, the B-cells were represented as real-valued vectors instead of bit-strings as its parent model, and the network affinity threshold (NAT) is introduced as a criterion to connect cells.

RLAIS (Resource Limited Artificial Immune System): In 2001 Timmis and Neal [24] modified the AINE model introducing the concept of Artificial Recognition Ball (ARB). An ARB is a representation of a family (type) of identical B-cells instead of a single B-cell. In the model, there is a resource pool (B-cells) with centralized control and the ARBs compete for allocating those resources. The interactions among self elements, the cloning and mutation processes are similar to those in AINE, but at the ARB level. Unlike AINE, those ARBs which have zero resources, are removed from the network, and the NAT is time independent and calculated from antigens data set.

SSAIS (Self-Stabilising Artificial Immune System): This model, presented by Neal [19] in 2001, is based on RLAIS for continuous analysis of time-varying data. The main difference between SSAIS and RLAIS, is that the former has no a limited resource level and each ARB can control its own level of resources (decentralized control). Additionally, SSAIS does not consider B-cell suppression in the calculus of stimulation level.

Meta-Stable Memory Immune Network: Following the AINE line, Neal [20] proposed a modified version of SSAIS in 2003 for data analysis, clustering and artificial immune memory. In this new model each ARB is stimulated by foreign antigens and by its neighbors in an Euclidean space like SSAIS. The main difference with SSAIS is that the system employs the cloning process only in a primary response, which is mediated by the NAT, but it does not consider mutation operator. All ARBs with a level of resources less than a fix mortality threshold are removed from the network.

Fractal Immune Network: Despite this model, proposed by Bentley and Timmis [2] in 2004, is not a modification of RLAIS, it uses the ARB concept and rename it Fractal Recognition Space (FRS). Here, interactions among self elements are considered to take place via artificial cytokines. Fractal cytokines are represented by a single clone of the transmitting FRS. The signal is received by a fractal receptor (a clone of the receiving FRS) and the distance is calculated (bitmap distance). If such distance falls below certain threshold, the transmitting FRS is stimulated only if it is a mature FRS. The immune network algorithm is based on
SSAIS. A stimulated FRS is cloned, with a fix probability, by creating a copy of the FRS and merged with the antigen following the merge-process of fractal proteins. If there exists no such a FRS, a new one is created at the antigen point as a primary response, like its parent model. Cellular death is concentrationdependent: each iteration, the FRS concentration is increased based on stimulation level. If the concentration drops bellow a mortality threshold, the element is removed from the network. The stimulation level of all FRSs in the system suffers a decay process that acts together with other operations as a population control mechanism.

Fuzzy AIS: in 2002, Nasraoui et al. [18] presented a model, based on RLAIS for performing clustering, web profiling and web mining. The Fuzzy ARB concept is introduced. A Fuzzy ARB defines a fuzzy set over the domain of discourse consisting of the training data set. Each fuzzy ARB is allowed to have its own scale/radius of influence (similar to NAT). Other difference with RLAIS is that those ARBs whose affinity is less than a certain threshold are merged (crossover operator). Stimulation and suppression interactions by both antigens and ARBs are considered. The cloning and mutation operators are applied over cells remaining after remotion (remove ARBs with zero B-cells allocated). The resource allocation process is modified.

TECNO-STREAMS: In 2003, Nasraoui et al. [17] adapted the Fuzzy AIS to perform stream data mining tasks. The self elements are named Dynamic Weighted B-Cell (D-W-B-cell), which defines an influence zone over the antigen space, as Fuzzy ARBs. However, since the data have a temporal aspect, the most current data have a higher influence than the older ones. The stimulation level not only decreases with the distance from the center, but with the elapsed time since the antigen was presented to the immune network. The affinity measure between cells is defined by the Robust Weight/Activation Function, which decreases with the Euclidean distance and the time factor of antigens, allowing the system to identify noisy data. The total stimulation that a D-W-B-cell perceived after certain number of antigens have been presented to the network, is given by the density of antigen population around the cell and the neighbor interactions. This process resembles the stimulation function of Fuzzy AIS, but considering the modified affinity measure. In order to decrease the number of network interactions, the immune network is separated in subnetworks so that the antigens disturb only one of such subnetworks. The network division is performed using the K-means algorithm.

\subsubsection{The aiNet Branch}

In 2001 de Castro and Von Zuben [7] proposed this model for data analysis tasks. It generates a network of antibodies linked according to the affinity (Euclidean distance). A subset of the antibodies with the highest affinity, with respect to a given antigen, is selected and cloned proportionally to the affinity. All generated clones are mutated inversely to its affinity. A fix percentage of clones is selected to be memory 
antibodies, by eliminating those whose affinity with the current antigen is less than a death threshold. If a pair of memory antibodies have an affinity greater than a suppression threshold, one of them is removed from the network. A remarkable difference between the aiNet branch and the Hunt \& Cooke branch is that aiNet does not consider the stimulation concept, but only the affinity concept. The training process of aiNet has a part inspired by the clonal selection principle and the other part uses antibody interactions for removing redundancy and finding data patterns.

Hierarchy of aiNets: de Castro and Timmis [6] in 2002 proposed a stopping criterion for aiNet algorithm based on Minimal Spanning Trees, and developed a technique for creating a Hierarchy of aiNets in order to find not only principal clusters in data sets, but subclusters. This is accomplished by structuring several networks in a kind of tree so that it is possible to separate automatically the clusters, and subclusters, found in training data sets.

opt-aiNet: As an adaptation of aiNet to multimodal function optimization, de Castro and Timmis [4] in 2002 proposed this model. The network cells interact accordingly with its affinity and by a suppression process that consists of removing those cells, which affinities are less than a fix threshold. All of the network cells undergo a cloning process by generating a fix number of copies. Those copies suffer mutation proportional to their parent's fitness. If the fitness error is significantly different from the previous iteration, the system performs the suppression process, and a fix percentage of new randomly generated cells are introduced into the network. Otherwise, the cells go on cloning and mutation processes.

IPD aiNet: Alonso et al. [1] make a modification of aiNet to model an agent that plays the Iterated Prisoner's Dilemma (IPD). In such a model, antigens and B-cells are represented as IPD strategies. An IPD strategy is considered as the way a player decides what move it will play given previous interactions. The immune agent perceives the opponent's strategy and try to find a strategy (most stimulated B-cell), in the immune memory, which provides it the highest payoff to confront the playing opponent. The main modification made to aiNet is in the memory mechanism: if a Bcell is added to memory it will never be removed.

\subsubsection{Other Models}

In 1994 Ishiguro [11] implemented an artificial immune network and demonstrated its potential applicability to a system of gait acquisition for a six-legged robot. Mitsumoto [16] extended this work by developing an environment with multiple robots. Despite the efficacy of these initiatives, the antibody interaction rules are generally defined in a rather ad hoc manner. The efficiency of this approach is restricted to low-complexity systems, which may be described using few antibodies [12].

Michelan \& Von Zuben: In 2002, Michelan and Von Zuben [15] presented an AIN model for autonomous control of mobile robot for multi-objective tasks. In this model, the antibodies represent an action the robot have to execute, and the antigens represent the current state of the robot. Stimulation and suppression interactions among antibodies are also modeled. The network dynamics is performed by genetic, crossover and mutation operators. An elitist selection operator is used in the training algorithm. The cellular death is performed by selecting those antibodies with a low fitness and replacing them by new generated antibodies.

CLARINET: CLARINET [26] is a model for autonomous navigation proposed by Vargas et al. in 2003. It is a hybrid non-parametric system which combines learning classifier systems, evolutionary algorithms and an artificial immune network. Classifier systems are regarded as B-cells, which interact each other with stimulation and suppression functions. The training algorithm considers crossover and mutation operators like as ones in the model proposed by Michelan et al. [15].

Reactive Immune Network: In 2004, Luh and Liu [14] proposed another model for autonomous navigation. As usual, the antigens are represented by states of the environment and antibodies are represented by the steering direction of the robot. The stimulation by antigens is calculated using the Artificial Potential Field approach, which considers a virtual attractive force between the robot and the target and a virtual repulsive force between the robot and obstacles. The stimulation and suppression among two antibodies is related to the difference between its steering angles.

AISEC (Artificial Immune System for E-mail Classification): The purpose of this model is interesting/uninteresting web information classification. The B-cells are represented by prototypes of uninteresting e-mail messages and the antigens are represented by the incoming email messages. If a B-cell is activated by an antigen, the message associated to it is labeled as uninteresting and sent to a special storage. The stimulation and suppression interactions are performed via increment and decrement of stimulation counter. Clonal selection process is performed and a B-cell can die via two death process: one is to eliminate those cells with stimulation counter equals zero, and the other one eliminates a B-cell when it makes a bad classification based on user feedback, this is, when the B-cell classification and the user classification do not match. This model was proposed by Secker et al. [23] in 2003.

\subsection{AIN Model Comparison}

This section presents a comparative analysis of the models described above. This analysis emphasizes specific aspects of the models such as B-cell/Antigen representation as well as affinity, stimulation and suppression measures.

Table 1 shows B-cell and antigen representation schemes used by different models. The most common representation scheme is an attribute list. Depending on the space of the problem, bit-strings or real-valued vectors are used. Each field represents a feature relevant to the problem; a bitstring field means the presence or absence of a particular feature, while a real-valued vector field represents a certain measure of the feature. Such a representation is adopted by Hunt \& Cooke and aiNet branches, except for Fuzzy AIS and TECNO-STREAMS, where the elements in $B$ contain not only the feature vector but its coverage radius. 
Table 1: Representation schemes for B-cells and antigens: bit-string and real-valued vector are the principal choice for representation. Other models use more complex representations in order to fit it to the particular application.

\begin{tabular}{|c|c|c|}
\hline Model & $B$ & $A$ \\
\hline Hunt \& Cooke & $\{0,1\}^{n}$ & $\{0,1\}^{n}$ \\
\hline AINE & $\Re^{n}$ & $\Re^{n}$ \\
\hline RLAIS & $\Re^{n}$ & $\Re^{n}$ \\
\hline SSAIS & $\Re^{n}$ & $\Re^{n}$ \\
\hline Meta-Stable IN & $\Re^{n}$, Fractal & $\Re^{3}$, Fractal \\
\hline Fractal IN & $\Re^{n} \times \Re$ & $\Re^{n}$ \\
\hline Fuzzy AIS & $\Re^{n} \times \Re$ & $\Re^{n} \times N$ \\
\hline TECNO-STREAMS & $\Re^{n}$ & $\Re^{n}$ \\
\hline aiNet & $\Re^{n}$ & $\Re^{n}$ \\
\hline Hierarchy of aiNets & $\Re^{n}$ & $\Re^{n}$ \\
\hline opt-aiNet & $I P D$ strategy & $I P D$ strategy \\
\hline IPD aiNet & $\{0,1\}^{n} \times S_{a c t i o n}$ & $\{0,1\}^{n}$ \\
\hline Michelan \& Von Zuben & $S_{t a g} \times\{0,1, \#\}^{n} \times\{0,1\}^{n}$ & $S_{t a g} \times\{0,1\}^{n}$ \\
\hline CLARINET & $\Re^{3}$ & $\Re^{3}$ \\
\hline Reactive IN & $S_{s b j} \times S_{\text {snd }}$ & $S_{\text {sbj }} \times S_{\text {snd }}$ \\
\hline AISEC & &
\end{tabular}

The Other Models branch has more complex representations. This group contains models created for autonomous navigation tasks and e-mail classification. In autonomous navigation models, like Michelan \& Von Zuben's model and CLARINET, the antigens represent states of the robot in the environment and the B-cells represent actions to be taken by the robot in the form of rules containing a condition to be satisfied $\left(\{0,1\}^{n}, S_{\text {tag }} \times\{0,1, \#\}^{n}\right)$, and the corresponding action $\left(S_{\text {action }},\{0,1\}^{n}\right)$. In AISEC, created for e-mail classification, the antigens represent the subject-sender tuples $\left(S_{s b j} \times S_{s n d}\right)$ of e-mails to be classified and B-cells represent subject-sender tuples $\left(S_{s b j} \times S_{s n d}\right)$ of uninteresting e-mails prototypes.

Notice that most of the models do not differentiate between phenotype and genotype of cells, except for Fractal IN, where phenotypes are represented by three real values, which are mapped into genotypes represented by Fractals. Additionally, the models use the same type of representation for both antigens and B-cells, except for TECNO-STREAMS, which consider a time factor as a part of the antigen representation; and the autonomous navigation models, where the antigens have no action factor in its representation.

In the models, the affinity measure is based on a distance measure in a certain problem-dependent space. The definition of an affinity measure implicitly generates a shapespace. As it is shown in Table 2, the two main ways to model affinity are: a function of the Hamming distance $\left(D_{H}\right)$, for bit-string representation, and a function of the Euclidean distance $(D)$, for real-valued vector representation. Notice that some models do not present explicitly the affinity function, but define the stimulation mechanism directly as $D$ or $1-D$. In general, a B-cell get stimulated by an antigen proportionally to its affinity with that antigen [22], the stimulation level depends on the affinity and, additionally, the stimulation level affects the cloning and mutation processes.

It is possible to classify the models, according to the cell interactions they model, in two groups: those that consider both stimulation and suppression interactions among
B-cells, and those that consider only stimulation interactions. SSAIS, Meta-Stable IN and Fractal IN conform the latter group. Regarding the suppression mechanism, the models in the aiNet branch, model the suppression as a killer or interchange operator, while the other ones model the suppression as a negative stimulation operator.

The effects of stimulation or suppression, experimented by a B-cell when interacts with another B-cell, are also affinity dependent. Some models consider the stimulation (and suppression) effect as a function of the affinity between two Bcells, others interpret suppression as a cellular death mechanism, and others simply do not consider the suppression effect.

Cloning and mutation processes constitute the principal mechanism for updating the network structure. According to immunology, B-cells undergo cloning and mutation if their total stimulation is greater than certain threshold [22]. Models mostly use that idea, although there are models that simply create a fix number of copies of the stimulated cell. Regarding mutation, there are models that perform it with a certain probability and others do it always, but consider a mutation rate calculated from the affinity or stimulation measure. As special cases, some models use genetic algorithms $(G A)$ as a mechanism to adapt the network structure.

\section{CONCLUSIONS}

In this paper, a general model of artificial immune network was presented. The model introduced a notation that served as a basis for a descriptive and comparative analysis of some AIN models. The relationships between models were shown in a genealogical tree of AIN models that has three main branches: The Hunt $\&$ Cooke branch, the aiNet branch and the Other Models branch.

The different models present immune concepts such as Bcells, antigens and B-cell/antigen interaction; and immune network theory concepts such as epitopes, paratopes and B-cell/B-cell interactions. There are also concepts that are 
Table 2: Modeling of affinity, simulation, suppression measures and cloning process: most of the measures are distance-based, and the number of clones created is, in general, proportional to the B-cell stimulation level. Empty cells mean that the corresponding function is not implemented by the model.

\begin{tabular}{|c|c|c|c|c|c|}
\hline Model & $f_{\text {affinity }}$ & $f_{\text {stimulation }}^{A}$ & $f_{\text {stimulation }}^{B}$ & $f_{\text {suppression }}^{B}$ & $f_{\text {cloning }}$ \\
\hline Hunt \& Cooke & $\sum_{k} G\left(D_{H}-\varepsilon+1\right)$ & $f_{\text {affinity }}$ & $f_{\text {affinity }}$ & $f_{\text {affinity }}$ & $k F$ \\
\hline AINE & $1-D$ & $f_{\text {affinity }}$ & $f_{\text {affinity }}$ & $f_{\text {affinity }}-1$ & $k F$ \\
\hline RLAIS & $1-D$ & $f_{\text {affinity }}$ & $f_{\text {affinity }}$ & $f_{\text {affinity }}-1$ & $k F$ \\
\hline SSAIS & $1-D$ & $f_{\text {affinity }}$ & $\frac{1}{n f_{\text {affinity }}}$ & - & $\frac{\sigma_{\text {decay }} R F}{10 \theta_{\text {mortality }}}$ \\
\hline Meta-Stable IN & $1-D$ & $\frac{1}{\frac{1}{f_{\text {affinity }}+2 D}}$ & $f_{\text {affinity }}+2 D+1$ & - & 1 \\
\hline Fractal IN & $1-D$ & $\frac{10 f_{\text {affinity }}}{\theta_{A M}}$ & $\frac{f_{a f f i n i t y}+2 D+1}{\theta_{C M}}$ & - & 1 \\
\hline Fuzzy AIS & $\exp \left(\frac{-D}{2 \sigma^{2}}\right)$ & $\frac{f_{\text {affinity }}}{\sigma^{2}}$ & $\frac{\alpha f_{a f f i n i t y}}{\sigma^{2}}$ & $\frac{-\beta f_{\text {affinity }}}{\sigma^{2}}$ & $k F$ \\
\hline TECNO-STREAMS & $\exp \left(-\left(\frac{D^{2}}{2 \sigma^{2}}+\frac{J}{\tau}\right)\right)$ & $\frac{f_{a f f i n i t y}}{\sigma^{2}}$ & $\frac{\alpha f_{a f f i n i t y}}{\sigma^{2}}$ & $\frac{-\beta f_{a f f i n i t y}}{\sigma^{2}}$ & $k F$ \\
\hline aiNet & $\frac{1}{D}$ & - & - & death & $k f_{a f f i n i t y}$ \\
\hline Hierarchy of aiNets & $\frac{1}{D}$ & - & - & death & $k f_{a f f i n i t y}$ \\
\hline opt-aiNet & $D$ & fitness & - & death & $k$ \\
\hline IPD aiNet & $D_{H}$ & - & - & death & $k f_{a f f i n i t y}$ \\
\hline Michelan \& Von Zuben & $\sum_{k} G\left(D_{H}-\varepsilon+1\right)$ & $f_{\text {affinity }}$ & $f_{\text {affinity }}$ & $f_{\text {affinity }}$ & $G A$ \\
\hline CLARINET & $\sum_{k} G\left(D_{H}-\varepsilon+1\right)$ & $f_{\text {affinity }}$ & $f_{\text {affinity }}$ & $f_{\text {affinity }}$ & $G A$ \\
\hline Reactive IN & - & $f_{\text {target }}+f_{\text {obstacle }}$ & $\cos \left(\theta_{i}-\theta_{j}\right)$ & $\cos \left(\theta_{j}-\theta_{i}\right)$ & $k F$ \\
\hline AISEC & $\frac{\text { words }}{\left|b_{\text {short }}\right|}$ & $s_{\text {counter }} \uparrow$ & $B-$ cell $\rightarrow M-$ cell & $s_{\text {counter }} \downarrow$ & $k F$ \\
\hline
\end{tabular}

modeled implicitly, for instance, the APCs (antigen presenting cells) are present in CLARINET: the model has some elements that receive antigens (messages) from the environment and present them to the network, which accomplishes the immune response. Finally, there are concepts that are not modeled at all, like the role of T-cells in the immune response.

In [21], some hypotheses and questions regarding immune network theory were outlined: (i) antigens modify the whole network or only a portion of it. (ii) the network can be seen either as a whole indivisible unit (open network) or as a set of nearly independent subnetworks (close network). (iii) there is some experimental evidence that if an antibody $a$ stimulates an antibody $b, b$ also stimulates $a$, but if the effect $a-b$ is suppressive, it is not clear how the $b-a$ effect is. Regarding (i), the Hunt \& Cooke model explores the idea of local antigen perturbation, it means that each antigen is presented to a subpopulation of the B-cells in the network. Regarding (ii), in the Hierarchy of aiNets model, this idea is implicitly used to separate the clusters and to find possible subclusters by constructing a network of aiNet connected subnetworks. However, this construction is not performed by an immunity-based approach. TECNO-STREAMS, also considers the whole system as a set of subnetworks, which are built using K-means, with the purpose of implementing a local antigen perturbation. Regarding (iii), some models of AIN use the mutual stimulation between B-cells, and others assume that if the B-cell $a$ stimulates the B-cell $b$ then $b$ suppresses $a$.

As an idea to create a population control mechanism, it could be useful to consider the fact that an antigen has a finite number of epitopes on its surface, it means that only a finite number of antibodies can effectively be stimulated by the antigen, namely, those which effectively bind to it. In the reviewed models, the antigen is presented to the whole network and every B-cell able to recognize it gets stimulated.
If only some of those B-cells are allowed to get stimulated, only those B-cells will suffer cloning and mutation.

\section{ACKNOWLEDGMENTS}

The authors would like to thank LISI (Intelligent Systems Research Lab., at National University of Colombia) members for their helpful comments and support.

\section{REFERENCES}

[1] O. M. Alonso, F. Nino, and M. Velez. A Robust Immune Based Approach to the Iterated Prisoner's Dilemma. In G. Nicosia, V. Cutello, P. J. Bentley, and J. Timmis, editors, Proceeding of the Third Conference ICARIS, pages 290 - 301, Edinburg, UK, September 2004. Springer.

[2] P. J. Bentley and J. Timmis. A Fractal Immune Network. In G. Nicosia, V. Cutello, P. J. Bentley, and J. Timmis, editors, Proceeding of the Third Conference ICARIS, pages 133 - 145, Edinburg, UK, September 2004. Springer.

[3] L. N. de Castro. Immune, Swarm, and Evolutionary Algorithms Part I: Basic Models. In ICONIP Conference (International Conference on Neural Information Processing). Workshop on Artificial Immune Systems 3, pages 1464 - 1468, 2002.

[4] L. N. de Castro and J. Timmis. An Artificial Immune Network for Multimodal Optimisation. In Congress on Evolutionary Computation. Part of the 2002 IEEE World Congress on Computational Intelligence, pages 699 - 704, Honolulu, Hawaii, USA, May 2002. IEEE.

[5] L. N. de Castro and J. Timmis. Artificial Immune Systems: A New Computational Intelligence Approach. Springer-Verlag, 2002.

[6] L. N. de Castro and J. Timmis. Convergence and Hierarchy of aiNet: Basic Ideas and Preliminary 
Results. In Proceedings of ICARIS (International Conference on Artificial Immune Systems), pages 231 - 240., University of Kent at Canterbury, September 2002. University of Kent at Canterbury Printing Unit.

[7] L. N. de Castro and F. J. V. Zuben. aiNet: An Artificial Immune Network for Data Analysis. In H. A. Abbas and R. A. S. andCharles S. Newton, editors, Data Mining: A Heuristic Approach, chapter XII, pages 231-259. Idea Group Publishing, USA, 2001.

[8] J. D. Farmer, N. H. Packard, and A. S. Perelson. The immune system, adaptation and machine learning. Physica, 22D:187 - 204, 1986.

[9] S. Garret. A Paratope Is Not an Epitope: Implications for Immune Network Models and Clonal Selection. In J. Timmis, P. Bentley, and E. Hart, editors, Proceedings of the Second International Conference ICARIS, pages 217 - 228, Edinburgh, UK, September 2003. Springer.

[10] J. E. Hunt and D. E. Cooke. Learning Using an Artificial Immune System. Journal of Network and Computer Applications, 19:189 - 212, 1996.

[11] A. Ishiguro, S. Ichikawa, and Y. Uchikawa. A Gait Acquisition of Six-Legged Robot Using Immune Networks. In Proceedings of International Conference on Intelligent Robotics and Systems (IROS '94), volume 2, pages 1034 - 1041, Munich, Germany, 1994.

[12] A. Ishiguro, T. Kondo, Y. Watanabe, Y. Shirai, and Y. Uchikawa. Immunoid: A Robot with a Decentralized Consensus-Making Mechanism Based on the Immune System. In ICMAS Workshop on Immunity-Based Systems, pages 82 - 92, December 1996.

[13] N. Jerne. Towards a Network Theory of the Immune System. Ann. Immunol. (Inst. Pasteur), 125C:373 389, 1974.

[14] G.-C. Luh and W.-W. Liu. Reactive Immune Network Based Mobile Robot Navigation. In G. Nicosia, V. Cutello, P. J. Bentley, and J. Timmis, editors, Proceeding of the Third Conference ICARIS, pages 119 - 132. Springer, 2004.

[15] R. Michelan and F. J. V. Zuben. Decentralized Control System for Autonomous Navigation Based on an Evolved Artificial Immune Network. In Proceedings of the IEEE Congress on Evolutionary Computation, volume 2, pages 1021 - 1026, Honolulu, HI, May 2002. IEEE.

[16] N. Mitsumoto, T. Fukuda, F. Arai, H. Tadashi, and T. Idogaki. Self-Organizing Multiple Robotic System. In Proceedings of the IEEE International Conference on Robotics and Automation, pages 1614 - 1619, Minneapolis, April 1996.
[17] O. Nasraoui, C. Cardona, C. Rojas, and F. González. TECNO-STREAMS: Tracking Evolving Clusters in Noisy Data Streams with a Scalable Immune System Learning Model. In Third IEEE International Conference on Data Mining, Melbourne, FL, November 2003. IEEE.

[18] O. Nasraoui, F. González, and D. Dasgupta. The Fuzzy Artificial Immune System: Motivations, Basic Concepts and Application to Clustering and Web Profiling. In IEEE International Conference on Fuzzy Systems, pages 711 - 716, Hawaii, HI, May 2002. IEEE.

[19] M. Neal. An Artificial Immune System for Continuous Analysis of Time-Varying Data. In J. Timmis and P. J. Bentley, editors, Proceedings of the 1st International Conference on Artificial Immune Systems (ICARIS), volume 1, pages $76-85$, University of Kent at Canterbury, September 2002. University of Kent at Canterbury Printing Unit.

[20] M. Neal. Meta-Stable Memory in an Artificial Immune Network. In J. Timmis, P. Bentley, and E. Hart, editors, Proceedings of the Second International Conference ICARIS, pages 168 - 180, Edinburg, UK, September 2003. Springer.

[21] G. Parisi. A Simple Model for the Immune Netwok. In Proceedings of the National Academy of Sciences of the United States of America, volume 87, pages 429 433, January 1990.

[22] A. S. Perelson and G. Weisbuch. Immunology for Physicists. Reviews of Modern Physics, 69(4):12191267, October 1997.

[23] A. Secker, A. Freitas, and J. Timmis. AISEC: An Artificial Immune System for E-mail Classification. In R. Sarker, R. Reynolds, H. Abbass, T. Kay-Chen, R. McKay, D. Essam, and T. Gedeon, editors, Proceedings of the Congress on Evolutionary Computation, pages 131 - 139, Canberra. Australia, December 2003. IEEE.

[24] J. Timmis and M. Neal. A Resource Limited Artificial Immune System for Data Analysis. Knowledge-Based Systems, 14:121 - 130, 2001.

[25] J. Timmis, M. Neal, and J. Hunt. An Artificial Immune System for Data Analysis. BioSystems, 55:143 - 150, 2000.

[26] P. A. Vargas, L. N. de Castro, R. Michelan, and F. J. V. Zuben. An Immune Learning Classifier System for Autonomous Navigation. In J. Timmis, P. Bentley, and E. Hart, editors, Proceedings of the Second International Conference ICARIS, pages $69-$ 80, Edinburg, UK, September 2003. Springer. 